\title{
INVENTORY OF SPECIES OF MILTOCHRISTA HÜBNER (LITHOSIINAE: ARCTIIDAE: LEPIDOPTERA) FROM NORTHWESTERN AND NORTHEASTERN INDIA
}

\author{
Amritpal S. Kaleka and H.S. Rose \\ Department of Zoology, Punjabi University, Patiala, Punjab 147002, India \\ Email: amritpalkaleka@indiatimes.com
}

\begin{abstract}
Inventory of sixteen species of Miltochrista Hübner, i.e. andamana Moore, arcuata Moore, conjuctana Walker, dharma Moore, euprepiodes Walker, frigida Walker, humilis Walker, ila Moore, linga Moore, nubifascia Walker, palmata Moore, punicea Moore, radians Moore, rubricosa Moore, strigipennis Herr. Schaffer and undulosa Walker of genus Miltochrista Hübner from various localities in North-west and Northeast India is given.
\end{abstract}

\section{Keywords}

Inventory, Lithosiin, Lepidoptera, Miltochrista, northeastern India, northwestern India, distribution

\begin{tabular}{l}
\multicolumn{2}{c}{ Abbreviations } \\
$\mathrm{Cu}_{1}-$ Upper cubital $\quad \mathrm{M}_{1}-\mathrm{M}_{3}-$ Median branches \\
$\mathrm{R}_{1}-\mathrm{R}_{5}-$ Radial branches \\
$\mathrm{Sc}+\mathrm{R}_{1}$ - First radial or second subcostal
\end{tabular}

\section{Introduction}

During the course of present investigations surveys were undertaken in different localities falling in the States of Arunachal Pradesh, Assam, Himachal Pradesh, Jammu and Kashmir, Manipur, Meghalaya, Sikkim and Uttaranchal for the collection of moth diversity referable to the subfamilies Arctiinae and Lithosiinae of the family Arctiidae. The representatives of subfamilies Arctiinae and Lithosiinae are commonly known as tiger and foot-man moths respectively. After proper sorting, followed by examination of various morphological characters such as the labial palpi, wing venation and maculation, as many as two hundred and forty eight specimens of sixteen species referable to a lithosiin genus Miltochrista Hübner have been identified from relevant literature (Hampson, 1894, 1900; Daniel, 1951, 1952; Watson et al., 1980; Fang, 1991). Accordingly, the present communication deals with an inventory of these species along with distribution and altitudinal range etc. The specimens are stored at the Department of Zoology, Punjabi University, Patiala, Punjab 147002, India.

\section{Material and Methods}

The adult moths were collected with the help of a portable light trap and from tube lights fitted at petrol pumps, forest and PWD rest houses. The specimens thus collected were stretched, pinned and preserved as per techniques in Lepidopterology. The morphological characters such as labial palpi, antennae, tibiae, wing venation and maculation have been studied from dried specimens for identification.

\section{Observations}

\section{Genus Miltochrista Hübner}

Hübner, [1819] 1816, Verz. Bekannter Schmett: 166

$$
\text { Type Species: Miltochrista miniata (Froster, 1771) }
$$

\section{Diagnosis}

Labial palpus short and porrect; antenna simple with cilia long in male, minute in female or bipectinated in male. Forewing with vein $R_{1}$ arising from one half to three-fourths length of cell, running along $S c$; veins $R_{3}-R_{5}$ stalked from angle of cell; $M_{1}$ originating from upper angle of cell or from the stalk of radial veins; $\mathrm{M}_{2}, \mathrm{M}_{3}$ and $\mathrm{Cu}_{1}$ approximated near lower angle of cell. Hindwing with vein $S c+R_{1}$ from middle of cell; $R_{5}$ and $M_{1}$ from upper angle of cell; $\mathrm{M}_{2}$ and $\mathrm{M}_{3}$ from lower angle or may be stalked. Legs with tibia having short spurs, mid-tibia with one pair and hind-tibia with two pairs of spurs. Male genitalia with uncus long, narrow and sickle-shaped; fenestrula rounded; tegumen almost of equal size of vinculum; vinculum U-shaped, saccus small; valva with sacculus and costa defined, saccular projection running along major portion of valva. Female genitalia with corpus bursae membranous, signum missing, ductus bursae 
guarded by genital plate, anterior apophysis shorter than posterior ones, papilla analis large with array of micro and macro setae.

\section{Distribution}

Palaearctic, Ethiopian, Oriental and Australian regions.

\section{Miltochrista andamana Moore}

Moore, 1877, Proc. Zool. Soc., 1877: 597.

Material examined: One male, 11.ix.1994, Bomdila, West Kameng District, Arunachal Pradesh, 2430m; one male, 23.ix.1994, one female, 24.ix.1994, two females, 27.ix.1994, Jatinga, District Haflong, North Cachar Hills, Assam, 900m; two females, one male, 18.ix.1994, Ukhrul, Manipur, 1060m; two females, 29.ix.1994, Jowaii, Jaintia Hills, Meghalaya, 670m; one male, 04.v.1995, Sikkim: Singtam, 600m; one male, 06.v.1995, Gangtok, 1660m; one female, one male, 08.v.1995, two males, 14.v.1995, Mangan, $1200 \mathrm{~m}$.

Wing expanse (half): Male: $12 \mathrm{~mm}$; Female: $12 \mathrm{~mm}$

Distribution: India: Andaman, Arunachal Pradesh, Assam, Manipur, Meghalaya, Sikkim.

Remarks: The species is reported for the first from northeastern India.

\section{Miltochrista arcuata Moore}

Moore, 1882, Lep. Ceylon. ii: 62; Lyclene rosea Hampson, 1891, Illust. Typical specimens Lep. Het. Colln. Br. Mus., viii: 50; Lyclene aurora Hampson 1891, Illust. Typical specimens Lep. Het. Colln. Br. Mus., viii: 50.

Material examined: One female, 18.ix.1994, Ukhrul, Manipur, 1060m; one male, 08.v.1995, Mangan, Sikkim, 1200m; one female, 05.v.1995, Gangtok, 1660m; one male, 15.vi.1994, Chakrata, Uttaranchal, $2100 \mathrm{~m}$.

Wing expanse (half): Male: $15 \mathrm{~mm}$; Female: $17 \mathrm{~mm}$.

Distribution: India: Manipur, Sikkim, Tamil Nadu (Nilgiris), Uttaranchal

Elsewhere: Sri Lanka

Remarks: All the above-mentioned localities are new records from northern and northeastern India.

\section{Miltochrista conjuctana (Walker)}

Conchylis conjuctana Walker, 1866, List specimens Lep. Insects Colln. Br. Mus., 35: 1788; M. tessellata Butler, 1881, III. Het., v :39.

Material examined: One female 11.ix.1991, one female, 24.ix.1994, two females, 26.ix.1994, one male, 27.ix.1994, one male, 28.ix.1994, one male, 28.ix.1995, Jatinga, District Haflong, North Cachar Hills, Assam, 900m; one female, 08.v.1995, Mangan, Sikkim $1200 \mathrm{~m}$.

Wing expanse (half): Male: $10 \mathrm{~mm}$; Female: $11 \mathrm{~mm}$

Distribution: India: Arunachal Pradesh, Assam, Nagaland, Sikkim Elsewhere: Bhutan

\section{Miltochrista dharma Moore}

Moore, 1879, Proc. Zool. Soc., 1879: 394.

Material examined: One female, 06.vii.1991, one female, 07.vii.1991, Solan, Himachal Pradesh, 1340m; one female, 28.vi.1995, Narakanda, 2700m; two males, 17.vi.2000, Sarahan, 1900m; two females, 29.ix.1994, one female, 30.ix.1994, Jowaii, Jaintia Hills, Meghalaya, 670m; one female, 06.vi.1993, one female, 07.vi.1993, Mossy falls, Mussorrie, Uttaranchal, 1645m; three females, 13.vi.1994, Kempty falls, 1524m; one female, 15.vi.1994, Chakrata, 2100m; one female, 10.vi.1995, Loharkhet, $1753 \mathrm{~m}$.

Wing expanse (half): Male: $22 \mathrm{~mm}$; Female: $24 \mathrm{~mm}$

Distribution: India: Northwestern Himalaya - Himachal Pradesh, Uttaranchal, Meghalaya, Nagaland (Karen Hills)

\section{Miltochrista euprepiodes (Walker)}

Hypocrita euprepiodes Walker, 1862, Journal Linn. Soc., VI: 102; Lyclene interserta Moore, 1878, Proc. Zool. Soc., 1878: 32. Material examined: Four females, one male, 23.ix.1994, one female, 26.ix.1994, three females, 28.ix.1994, one female, two males, 10.ix.1995, one female, one male, 27.ix.1995, two males, 29.ix.1995, one male, 30.ix.1995, one female, one male, 01.x.1995, Jatinga, District Haflong, North Cachar Hills, Assam, 900m.

Wing expanse (half): Male: $15 \mathrm{~mm}$; Female: $16 \mathrm{~mm}$

Distribution: India: Arunachal Pradesh, Assam, Sikkim, Nagaland.

Elsewhere: China, Myanmar, Borneo, Malaysia, Philippines. Remarks: Jatinga is the new distributional record for this species from Cachar Hills, Assam.

\section{Miltochrista frigida (Walker)}

Doliche frigida Walker, 1854, List specimens Lep. Insects Colln. Br. Mus., 2: 530; Lyclene diffusa Walker, 1862, Journ. Proc. Linn. Soc., VI: 111

Material examined: Two females, 11.ix.1991, one female, 27.ix.1994, three females, three males, 28.ix.1995, two males, 30.ix.1995, Jatinga, District Haflong, North Cachar Hills, Assam, 900m; one male, 14.v.1995, Mangan, Sikkim, 1200m.

Wing expanse (half): Male: $11 \mathrm{~mm}$; Female: $13 \mathrm{~mm}$

Distribution: India: Arunachal Pradesh, Assam, Sikkim, Nagaland Elsewhere: China, Borneo, Malaysia, Myanmar.

\section{Miltochrista humilis Walker}

Walker, 1854, List specimens Lep. Insects Colln. Br. Mus., 2: 544; Setina calamaria Moore, 1888, Proc. Zool. Soc., 1888: 392; Setina nebulosa Moore, 1878, Proc. Zool. Soc., 1878: 35; Setina dasara Moore, 1857, Cat. Lep. Insects Mus. Hon. E.I.Co: 303. Material examined: One female, 30.ix.1995, three females, 01.x.1995, Jatinga, District Haflong, North Cachar Hills, Assam, $900 \mathrm{~m}$.

Wing expanse (half): Male: Not examined; Female: 10mm Distribution: India: Assam, Nagaland, Sikkim, Punjab, Tamil Nadu (Nilgiris). 
Remarks: Jatinga is its new distributional record from Cachar Hills, Assam.

\section{Miltochrista ila Moore}

Moore, 1857, Cat. Lep. Mus. Hon.E. I.Co., 1857: 300; id. 1882, Lep. Ceylon. ii: 103.

Material examined: One male, one ?, 02.vii.1994, Kainchi Dham, Uttaranchal, 1800m; two females, 03.vii.1994, Ranikhet, 1830m. Wing expanse (half): Male: $13 \mathrm{~mm}$; Female: $14 \mathrm{~mm}$

Distribution: India: Canara, Uttaranchal

Elsewhere: Sri Lanka

Remarks: The species collected for the first time from the above localities in northern India.

\section{Miltochrista linga (Moore)}

Barsine linga Moore, 1857, Cat. Lep. Insects Mus. Hon. E. I. Co., 1857: 301.

Material examined: One female, 26.ix.1994, Jatinga, District Haflong, North Cachar Hills, Assam, 900m; two females, 06.vii.1991, one female, one male, 26.ix.1991, Solan, Himachal Pradesh, 1340m; one male, 02.viii.1994, Nauni, 1369m; four females, 07.ix.1995, twelve females, 08.ix.1995, Dharamshala, 1200m; five females, five males, 09.ix.1995, Macleodganj, 1800m; three males, 12.vi.2000, Kotkhai, 1526m.

Wing expanse (half): Male: $16 \mathrm{~mm}$; Female: $22-23 \mathrm{~mm}$.

Distribution: India: Assam, Himachal Pradesh, Sikkim.

Elsewhere: China.

Remarks: The species is being reported for the first time from Jatinga Assam.

\section{Miltochrista nubifascia (Walker)}

Barsine nubifascia Walker, 1863, List specimens Lep. Insects Colln. Br. Mus., 31: 251; Butler, 1881, Illust. Typical specimens Lep. Het. Colln. Br. Mus., v: 86; Barsine punctifascia Walker, 1869, Char. Undeser. Het: 9; Lyclene indistincta Moore, 1878, Proc. Zool. Soc., 1878: 33.

Material examined: One female, two males, 10.ix.1994, Bomdila, West Kameng District, Arunachal Pradesh, 2430m; two males, 12.ix.1994, Dirang, 1497m.

Wing expanse (half): Male: $13-15 \mathrm{~mm}$; Female: $14 \mathrm{~mm}$

Distribution: India: Arunachal Pradesh, Sikkim

Elsewhere: China

Remarks: The collection of the species from Arunachal Pradesh is its first record from northeastern India.

\section{Miltochrista palmata Moore}

Moore, 1878, Proc. Zool. Soc., 1878: 31

Materials examined: One male, 03.x.1995, Bomdila, West Kameng District, Arunachal Pradesh, 2430m; one male, 13.ix.1991, one male, 25.ix.1995, two males, 29.ix.1995, two males, 30.ix.1995, Jatinga, District Haflong, North Cachar Hills, Assam, 900m; five females, 30.viii.1994, Ramnagar, Jammu \& Kashmir, 1206m.

Wing expanse (half): Male: 14mm; Female: $16 \mathrm{~mm}$
Distribution: India: Arunachal Pradesh, Assam, Himachal Pradesh, Jammu and Kashmir.

Elsewhere: China.

Remarks: Ramnagar is the new distributional record from Jammu and Kashmir.

\section{Miltochrista punicea (Moore)}

Barsine punicea Moore, 1878, Proc. Zool. Soc., 1878: 29

Material examined: Two females, 10.ix.1991, one female, 25.ix.1994, one female, 27.ix.1994, two females, 28.ix.1994, two males; 28.ix.1995, one female, two males, 01.x.1995, Jatinga, District Haflong, North Cachar Hills, Assam, 900m; two females, 14.v.1995, Mangan, Sikkim, 1200m.

Wing expanse (half): Male: $10 \mathrm{~mm}$; Female: $13 \mathrm{~mm}$

Distribution: India: Arunachal Pradesh, Assam, Sikkim

Elsewhere: China, Bhutan, Myanmar

Remarks: The availability of this species from Assam is its new distributional record.

\section{Miltochrista radians (Moore)}

Lyclene radians Moore, 1878, Proc. Zool. Soc., 1878: 30

Material examined: One male, 03.x.1995, Bomdila, West Kameng District, Arunachal Pradesh, 2430m; one male, 26.ix.1994, one male, 27.ix.1995, two females, 01.x.1995, Jatinga, District Haflong, North Cachar Hills, Assam, 900m; two females, 13.viii.1991, Solan, Himachal Pradesh, 1340m; two males, two females, 15.vi.2000, Sangla, 2680m; two females, 16.vi.2000, Recongpeo, $2400 \mathrm{~m}$.

Wing expanse (half): Male: 10-13mm; Female: $12-15 \mathrm{~mm}$

Distribution: India: Arunachal Pradesh, Assam, Himachal Pradesh, West Bengal (Calcutta)

Elsewhere: China

Remarks: All the above-mentioned localities are new distributional records from northern and northeastern India.

\section{Miltochrista rubricosa (Moore)}

Lyclene rubricosa Moore, 1878, Proc. Zool. Soc., 1878: 30; Lyclene curvifascia Hampson, 1891, Illust. Typical specimens Lep. Het. Colln. Br. Mus., viii: 49; Lyclene ochracea Hampson, 1891, Illust. Typical specimens Lep. Het. Colln. Br. Mus., viii: 51. Material examined: Two females, 11.vi.1994, two females, 12.vi.1994, Mussorrie, Uttaranchal, 2030m.

Wing expanse (half): Male: Not examined; Female: $14 \mathrm{~mm}$ Distribution: India: Uttaranchal, Maharastra (Bombay), Tamil Nadu (Nilgiris)

Elsewhere: China; Sri Lanka.

Remarks: The aforesaid locality is a new record from Western Himalaya.

\section{Miltochrista strigipennis (Herrich Schäffer)}

Paidia strigipennis Herrich Schäffer, 1843, Samml. Aussereur. Schmett: 437; Lyclene discistriga Moore, 1878, Proc. Zool. Soc., 1878: 32; Lyclene terminata Moore, 1878, Proc. Zool. Soc., 1878: 
33; Lyclene sinica Moore, 1877, Ann. Mag. Nat. Hist., (4) xx: 87; Lyclene inconsticiua Moore, 1878, Proc. Zool. Soc., 1878: 32. Material examined: One female, 23.ix.1994, one female, 25.ix.1994, four females, two males, 26.ix.1994, one male, 27.ix.1994, Jatinga, District Haflong, North Cachar Hills, Assam, 900m; two males, 30.vi.1995, nine males, 17.vi.2000, Sarahan, Himachal Pradesh, 1900m; one male, 17.ix.1994, Chakhabama, Nagaland, 1212 m; one female, 04.v.1995, Singtam, Sikkim, 600m; one male, 06.v.1995, Gangtok, 1660m; one male, 08.v.1995, Mangan, 1200m; one male, 12.vi.1994, Mussorrie, Uttaranchal, 2030m; one female, one male, 08.vi.1995, Choukari, 2010m; one male, 09.vi.1995, ten males, 10.vi.1995, Loharkhet, 1753m; two males, 11.vi.1995, Song, 1500m; two males, 12.vi.1995, Kasauni, 1800m.

Wing expanse (half): Male: 18mm; Female: $22 \mathrm{~mm}$

Distribution: India: Arunachal Pradesh, Assam, Himachal Pradesh, Nagaland, Sikkim, Uttaranchal

Elsewhere: China, Bhutan, Myanmar, Sumatra

Remarks: The collection of this species from the Western Himalayas is its first report.

\section{Miltochrista undulosa (Walker)}

Cyllene undulosa Walker, 1854, List specimens Lep. Insects Colln. Br. Mus., 2: 545; Miltochrista straminea Walker, 1856, List specimens Lep. Insects Colln. Br. Mus., 7: 1685; Sesapa decurrens Walker, 1864, List specimens Lep. Insects Colln. Br. Mus., 31: 255; Sesapa excurrens Walker, 1864. List specimens Lep. Insects Colln. Br. Mus., 31: 255.

Material examined: One female, 10.ix.1991, one female, 13.ix.1991, one male; 14.ix.1991, Jatinga, District Haflong, North Cachar Hills, Assam, 900m; eleven males, seven females, 12.vi.2000, Kotkhai, Himachal Pradesh, 1526m; one female, Bhali, 2400m; one female, Jeouri, 2200m; five females, four males, Sarahan, 1900m; six males, 29.vi.1994, two females, two males, 30.vi.1994, Nainital, Uttaranchal, 1938m

Wing expanse (half): Male: $12 \mathrm{~mm}$; Female: $14 \mathrm{~mm}$
Distribution: India: Arunachal Pradesh, Assam, Himachal Pradesh, Sikkim, Uttaranchal

Elsewhere: China; Bhutan; Myanmar.

\section{Acknowledgement}

Dr. Kaleka is thankful to C.S.I.R., New Delhi and Dr. Rose to Ministry of Environment and Forests, New Delhi for financial assistance.

\section{References}

Arora, G.S. and M. Chadhury (1982). On the lepidopterous fauna of Arunachal Pradesh and adjoining areas of Assam in North-East India: Family Arctiidae. Zoological Survey of India- Technical Monograph 6: 1-66.

Daniel, F. (1951). Beitrage zur Kenntnis der Arctiidae Ostasiens under besonderer Beriicksichtigung der Ausbeuten. H. Hones aus diesens Gebiets Lepidoptera Heterosera. Part III Lithosiinae 2 (3-4): 291-327.

Daniel, F. (1953). Beitrage zur Kenntnis der Arctiidae Ostasiens under besonderer Beriicksichtigung der Ausbeuten. H. Hones aus diesens Gebiets Lepidoptera Heterosera. Part III Lithosiinae 3(1-2): 75-90.

Fang, C. (1991). Studies on genus Miltochrita of China (Lepidoptera: Arctiidae: Lithosiinae). Sinozoologia 0(8): 383-395.

Hampson, G.F. (1894). Fauna of British India, including Ceylon and Burma, Moths. Taylor and Francis Ltd., London, 690pp.

Hampson, G.F. (1900). Catalogue of the Lepidoptera Phalaenae in the British Museum 2: 1-590.

Watson, A., D.S. Fletcher and I.W.D. Nye (1980). The generic names of the moths of world. Noctuoidea 2: 1-228 